\title{
Erziehung als Handwerk oder industrielle Massen- anfertigung? Wider die Heraufbeschwörung simpler Gegensätze und Allegorien
}

\section{Esther Berner}

Die vorliegende Replik entfaltet ihre Kritik und Gegenposition ausgehend von zwei zentralen, in den Augen der Autorin jedoch unzutreffenden Argumentationen Herzogs: Jener stellt in seinem Beitrag die Vorstellung von Erziehung als Handwerk der Erziehung als industrielle Massenanfertigung entgegen. In diesem Beitrag wird eine alternative Betrachtung der zugrunde liegenden Normierungsfrage vorgeschlagen. Diese - für die Schule alles andere als neue - Frage sollte, so der Vorschlag, nicht apodiktisch, sondern nach Massgabe einer Balance zwischen dem Anspruch der Gerechtigkeit und der Akzeptanz von Differenz angegangen werden. Einhergehend mit der beklagten Normierungstendenz sieht Herzog die Gefahr einer Deprofessionalisierung des Lehrberufs. Dem wird ein gemessen am öffentlichen Auftrag der Lehrpersonen realistisches und der Profession selbst dienliches Berufsverständnis entgegengehalten. Basis der der Lehrerschaft vom Autor zugesprochenen Autonomie wäre hier nicht das von ihm eingeforderte Vertrauen der "Abnehmer" und "Auftraggeber", sondern die Idee der geteilten Verantwortung.

Walter Herzog provoziert in der vorliegenden Kritik an den laufenden Bildungsreformen mit der Herleitung von Funktion und Zweck schulischer Standards von der Anfertigung von Maschinengewehren. Herzog gehört zu den Erziehungswissenschaftlern, die um die Wirkungsmacht pädagogischer Sprachen im Positiven und Negativen wissen (Herzog, 2002), umso ernster muss man die in diesem Zusammenhang von ihm benutzten Allegorien - Handwerk vs. industrielle Massenanfertigung - wohl nehmen. Zugleich evoziert er auf diese Weise eine «alte» Frage, nämlich diejenige nach dem angemessenen professionellen Verständnis des Lehrberufs. Ich werde diesen Problemzusammenhang in meinem Kommentar im zweiten Abschnitt berühren; vorerst scheint es mir aber notwendig, vor dem Hintergrund jener Einordnung aktueller Schulreformkonzepte in den Bereich industrieller (Waffen-)Fertigung die zentralen Konzepte «Bildungsstandards' " und neue "Bildungssteuerung» in klärender Absicht nochmals aufzugreifen. Die Vorlage für den dritten und letzten Punkt meiner Replik bietet Herzogs Hinweis auf grundsätzliche theoretische Unzulänglichkeiten, die 
dem aktuellen Reformkonzept implizit zugrunde liegen. Hier sei die Frage erlaubt, inwiefern seine eigene Argumentationsweise die von ihm geforderten wissenschaftlichen Gütekriterien einzulösen vermögen.

\section{Die HarmoS-Standards: inwiefern ein Normierungs- instrument?}

Es fällt auf, dass Herzog in seiner Kritik an Bildungsstandards an keiner Stelle erwähnt, dass es sich bei den Schweizer Festlegungen um Mindeststandards handelt. Tatsächlich verliert aber das heraufbeschworene Normierungsszenario einiges an Wirkung, wenn man diese Spezifizierung vornimmt. Um Bekanntes an dieser Stelle zu wiederholen: Es kann sich bei der Idee von Mindeststandards kaum darum handeln, das Lernen der Schülerinnen und Schüler auf ein solches Minimum hin zu normieren. Definiert und insofern normiert oder, gefälliger ausgedrückt: harmonisiert werden aber tatsächlich in gewissen Leistungsfächern und auf dem, wie es der Terminus besagt, kleinsten Nenner Mindestleistungen; es handelt sich damit um Kompetenzen, über die jede Schülerin und jeder Schüler auf einer gewissen Klassenstufe unabhängig von der besuchten Schule verfügen soll. Dies ebenso wie eine gewisse strukturelle Angleichung der kantonalen Schulsysteme unter HarmoS wird von den Eltern und - wie die Abstimmung vom Mai vorletzten Jahres zur Revision der Bildungsartikel in der Bundesverfassung deutlich gezeigt hat - vom Grossteil der Bürger/innen als wünschenswert betrachtet. Die Angleichung von Mindestlernanforderungen hat auf Schülerseite aber auch mit Gerechtigkeit zu tun - ich werde auf diesen Aspekt nochmals zurückkommen -, und im besten Fall darf man die Leistungsanforderungen im Sinne eines Bildungsauftrages verstehen.

Auf einen Irrtum Herzogs trifft man an der Stelle, wo er behauptet, bei den Schweizer Bildungsstandards handle es sich im Gegensatz zu den herkömmlichen Steuerungsmitteln, Gesetzen, Lehrplänen, Reglementen etc., um «Produktionsanleitungen, die den Fertigungsprozess [Lehr- und Lernprozesse, E.B.] im Detail regeln»; es sind vor allem diese «Fertigungsprozesse» und damit die Unterrichtsmethoden, die Bildungsstandards in seinem Verständnis zu steuern und zu normieren beabsichtigen. Nun widersprechen jene Zuschreibungen aber nicht nur der massgeblichen Definition von Bildungsstandards und deren Funktion in der Expertise von Klieme et al. (2003); selbst in der von ihm angeführten Literatur aus dem Umkreis der EDK findet man diese Behauptung so nicht. Vielmehr liest man dort, dass es sich bei den Schweizer Bildungsstandards um Leistungsstandards handelt, deren Normen «nicht auf Lerninhalte (content standards) und nicht auf vorhandene schulische Ressourcen (opportunity-to-learnstandards)" ausgerichtet sind (Maradan \& Mangold 2005, S. 4).

In Herzogs Argumentation dominieren eindeutig die Befürchtungen einer Normierung des schulischen Unterrichts; umso überraschender ist, dass er an anderer Stelle eine dem Kompetenzkonzept inhärente Gleichgültigkeit gegen- 
über Inhalten und Methoden beklagt. Nun ist es aber falsch anzunehmen, dass «Kompetenzen», wie sie den (nationalen) Bildungsstandards zugrunde liegen, nichts mit «Wissen» zu tun haben - ein Irrtum Herzogs, vor dem ihn eigentlich die Expertise von Klieme et al. (2003, S. 20), die zu der von ihm selber angeführten Referenzliteratur zählt, bewahren müsste.

Semantisch eignet sich der Begriff Bildungsstandards vorzüglich für eine Kritik, die den Normierungscharakter aktueller Reformvorhaben fokussiert. Eigentlich legen Standards im Bildungsbereich je nach Definition aber «nur» fest, wie sich ein von sämtlichen Schülerinnen und Schülern nicht zu unterschreitendes Minimum (Mindeststandards), ein erwarteter Durchschnitt (Regelstandards) oder das Optimum (Maximalstandards) einer bestimmten fachlichen Kompetenz bestimmen. Interessanter für die Analyse ist deshalb das Konstrukt «Kompetenzmodell», das den Fächerstandards zugrunde liegt. Diese sind es nämlich, denen eine inhaltlich normierende Funktion zukommt. Insofern könnte man tatsächlich der von mir weiter oben zitierten offiziellen Definition der HarmoSStandards als «reine» Leistungsstandards entgegenhalten, dass solche kaum abgelöst von inhaltlichen Dimensionen definiert werden können. Im Fach Mathematik etwa kommen diese auf der Ebene der «Leitideen» zum Tragen, die allerdings nicht mit den klassischen Stoffgebieten verwechselt werden sollten. Abgesehen davon hängt das Ausmass einer allfälligen Normierungswirkung in erster Linie davon ab, ob bzw. inwieweit Kompetenzmodelle als Referenz für (Rahmen-)Lehrpläne, Lehrmittel, Schülerleistungstests, Selektionsprüfungen etc. herangezogen werden sollen. Diesbezüglich befindet sich der «Laie» allerdings noch immer im Unklaren, was die EDK gerne damit begründet, dass es die Kantone und allenfalls Regionen sind, die hierüber zu entscheiden haben. Es ist diese Ungewissheit bezüglich bildungspolitisch eminent bedeutsamer Weichenstellungen, die der kritischen Diskussion auf einem notwendig differenzierten Niveau enge Grenzen setzt. Diese Bemerkung leitet über zum anderen zentralen Kritikpunkt Herzogs, der sich mit dem Stichwort «Deprofessionalisierung» benennen und um die Frage der «Legitimierung» erweitern lässt.

Es mussten nicht erst Bildungsstandards bzw. Kompetenzmodelle erfunden werden, um schulische Unterrichtsinhalte und Lernziele festlegen und gewissermassen normieren zu können, wenngleich dies in der Geschichte der obligatorischen Volksschule noch nie auf gesamtschweizerischer Ebene geschah. Umgekehrt muss man an das Zustandekommen der Kompetenzmodelle dieselben Fragen stellen wie an die Erarbeitung traditioneller Lehrpläne: Wie legitimieren sich Entscheide hinsichtlich der Gewichtung eines Faches und seiner Komponenten? Welche umfassenderen Bildungsziele stehen dahinter? Und wer - in personeller wie professioneller Hinsicht - fällt die diesbezüglichen Entscheide? Lehrplanentwicklung zeichnet sich in der Schweiz bisher durch eine insgesamt geringe Spezialisierung aus. Sie erfolgt nach den Gebräuchen des gängigen Milizsystems unter breiter Beteiligung von Schulpraktikerinnen/Schulpraktikern und der Verwaltung (Seliner-Müller, Fries \& Künzli, 1999, S. 33). Zudem 
durchlaufen kantonale Lehrpläne vor der Inkraftsetzung gewöhnlich eine breite Vernehmlassung und (Teil-)Erprobung; eine langfristige Entwicklungsdauer ist daher die Regel. Demgegenüber ist die Umsetzung des neuen Steuerungsparadigmas in starkem Mass auf wissenschaftliche Fachexpertise angewiesen; die Entwicklung von Kompetenzmodellen und Tests gehört weitestgehend in den Zuständigkeitsbereich von Spezialistinnen/Spezialisten aus den Fachdidaktiken und der (pädagogischen) Psychologie. Es liegt auf der Hand, dass diese Verschiebung auf der Akteursseite im Zeichen einer Spezialisierung und Rationalisierung mit einer Abnahme von Legitimation und damit verbunden unter Umständen von Akzeptanz einhergeht. Zudem finden auf dem Weg bis zur Verabschiedung der HarmoS-Standards durchaus einige Vernehmlassungen und Konsultationen statt; die Verfahren laufen allerdings auf einer verhältnismässig distalen Ebene ab. Die Kantone, welche dem Konkordat beitreten und z.T. bereits beigetreten sind, verpflichten sich im Übrigen automatisch zur Einhaltung der Mindeststandards in der Formulierung, die später, voraussichtlich im Herbst dieses Jahres, von der EDK-Plenarversammlung verabschiedet und dem Konkordat als Anhang beigefügt werden.

\section{Handwerk vs. industrielle Fertigung}

Herzog greift in der Kritik an den gegenwärtigen Tendenzen in Bildungspolitik und -forschung in provokativer und zugleich simplifizierender Weise auf die Gegenüberstellung industrieller Fertigungsprozeduren einerseits und handwerklicher Herstellungsverfahren anderseits zurück. Erstere stehen dabei in Analogie zu einem technologischen Erziehungsverständnis, wie es in seinen Augen die Politik, unterstützt von wissenschaftlichen "Agenten», verkörpert. Diesen wird dabei eine Sicht auf Erziehung und Bildung unterstellt, die «output» als ein durch die Setzung von Zielnormen steuerbares, das heisst kausal herbeiführbares Ergebnis versteht. Nun ist im Kontext der Rede von einer neuen output-orientierten Steuerung zwar tatsächlich die Idee der Sichtbarmachung von Ergebnissen im Sinne der Evidenz leitend. Unterschlagen wird in Herzogs Darstellung aber eine weitere Komponente des Konzeptes, jene Feedback-Schleife nämlich, mittels derer Ergebnis-Daten zu einer Grundlage von Steuerungsentscheiden, die sich mit auf den input wie auf Prozesse beziehen können, werden sollen. «Der Knackpunkt einer outputorientierten Steuerung» besteht nach Oelkers und Reusser (in Druck, 2008 , S. 211) denn auch gerade in dieser «Rückkoppelung von durch Tests und andere Evaluationsverfahren ermittelten Lernergebnissen in schulbezogene Entwicklungsmassnahmen [...]».

Ist im Zitat von Evaluationsverfahren die Rede, so darf man bezüglich der Notwendigkeit des Einbezugs von Kontextdaten von einem breiten wissenschaftlichen Konsens ausgehen - wie sonst sollten sinnvolle Aussagen und Thesen in diesem Feld generierbar sein? Damit sind wir allerdings unweigerlich im 
Gebiet wissenschaftlich-methodologischer Techniken angelangt. Die von Herzog verwendete Sprache verleiht entsprechenden Verfahren leicht den Anstrich inhumaner Reduktion, und deshalb bevorzugt er ein Verständnis von Erziehungs- und Unterrichtsprozessen, das sich mit demjenigen der «handwerklichen Produktion» vergleichen lässt: Denn «[n]icht lokales Einpassen wie beim Handwerk, sondern kontextfreies Messen ist die Basis industrieller Fertigung» [Hervorhebung i.O.]; oder: "Wie für industrielle Standards, [sic] ist für Bildungsstandards die Überwindung der Beschränkung auf lokale Vergleiche charakteristisch.» Man mag sich nun fragen, was mit diesen lokalen Bedingungen gemeint sein könnte, deren Berücksichtigung der Autor fordert. Der Kontext der Äusserungen legt die Bezugnahme auf den schulischen Gegenstandsbereich von Notenvergabe und Selektion nahe: Handelt es sich bei Herzogs Rede also tatsächlich um ein Plädoyer für die gängige, am Klassendurchschnitt orientierte Benotung? Es käme allerdings einer Bankrotterklärung der öffentlichen Schulen in der Schweiz gleich, wenn ein Vorzug darin gesehen würde, dass Mindestanforderungen in Fächern wie Mathematik, Naturwissenschaften, Erst- und Fremdsprache(n) sich in der einen Schule, sagen wir in der Stadt Zürich, von der andern, sagen wir in einer Walliser Bergregion, aus Gründen lokaler Anpassung unterscheiden. Um den Nachweis für die Problematik solch eines Ideals zu erbringen, brauchen wir allerdings kaum eine neue Bildungstheorie als Ergebnis kritischer Emanzipation der Pädagogik von Anliegen der Politik, vielmehr reicht der Rückgriff auf die etablierte ethische Kategorie «Gerechtigkeit».

Hat Gerechtigkeit hier mit Normen, nämlich der Gleichheit der Bezugsnormen von Beurteilungen zu tun, ist auf der anderen Seite die Frage, inwiefern (standardisierte) Tests systematisch als Referenz für Notenvergabe und Selektion beigezogen werden sollen, tatsächlich eine professionspolitisch heikle und bedarf einer ernsthaften Diskussion. Immerhin belehrt einen der Blick auf Erfahrungen im Ausland, und hierbei ganz besonders auf England, dass vermehrte Kontrolle und Transparenz bezüglich Schulleistungen und ihres Zustandekommens nicht unbedingt zu mehr Vertrauen in ein Bildungswesen und die Arbeit der Lehrerprofession führen (Wolf, 2000). «Vertrauen», wie es Herzog fordert, lässt sich ganz offensichtlich nicht einfach voraussetzen oder herstellen. Angemessener scheint mir deshalb der Rückgriff auf den weniger emphatischen und besser rationalisierbaren Begriff der «Verantwortung», und zwar der «geteilten Verantwortung». Verantwortung für das Lernen von Schülerinnen und Schülern und die Qualität der Schulen sollte auch, aber gerade unter der Voraussetzung eines öffentlich-staatlichen Volksschulwesens nicht einseitig den Lehrkräften zugeschrieben werden. «Verantwortung» scheint mir zudem in sinnvollerer Weise an den anderen von Herzog eingebrachten Begriff, denjenigen der Autonomie, anknüpfbar; schliesslich setzt das Zugeständnis von Freiheit nicht nur Vertrauen voraus, wie dieser es ausdrückt, sondern auch Verantwortung. Und genau deshalb ist ein Mehr an Autonomie nicht immer nur entlastend, zumindest solange wir es bei den Volksschullehrern nicht mit zünftischen Handwerkern oder freien 
Künstlern zu tun haben. Volksschullehrer/innen stellen per definitionem auch keine Professionisten dar. Vielmehr handelt es sich bei diesem Beruf um eine Semiprofession, und zwar gerade weil das Kriterium der "Autonomie gegenüber Klienten und Staat» nicht gegeben ist. Dass dies so ist, hat seine historischen Gründe (Bloch Pfister, 2007) und ist deshalb konstitutiv für unser Verständnis von öffentlich-staatlichem Unterricht. Wie das Gleichgewicht zwischen Autonomie und Verantwortung auf der Ebene der Lehrpersonen und Einzelschulen auch in Zukunft, das heisst im Zeichen laufender Schulreformen, zu erhalten ist, wird vor diesem Hintergrund zur zentralen Frage. Problematisch und geradezu fatal erscheint mir aber der Appell an das "Vertrauen» in die Arbeit der Lehrpersonen, wenn er, wie im Fall Herzogs, mit einer grundlegenden Skepsis gegenüber der Steuerbarkeit und Rationalisierbarkeit von Lehr- und Lernprozessen gepaart ist.

\section{Evidenzen, Argumente - und Werte}

Ich komme zum Schluss und damit zu einigen zusätzlichen Überlegungen betreffend die argumentative Qualität von Herzogs Beitrag. Meine Skepsis bezüglich der Produktivität der vom Autoren verwendeten sprachlichen Mittel habe ich bereits zum Ausdruck gebracht. Ich möchte diese durch den Eindruck ergänzen, dass wir in der Diskussion durch ein solches Denken in einfachen Gegensätzen hinter die erreichte Differenziertheit des Wissensstandes hinsichtlich der Wirkung von Bildungsstandards zurückfallen. Ich füge dies deshalb an, weil uns Herzog selber daran erinnert, dass nicht «Evidenzen» "[d] en Kern der wissenschaftlichen Rationalität bilden», «sondern Argumente» [Hervorhebung E.B.]. Nun tragen Begriffe wie «evidence-based policy» tatsächlich die Gefahr positivistischer Kurzschlüsse in sich. Die Anwendung solcher Begrifflichkeiten auf einen Bereich, der es im Kern mit Prozessen der Bildung und deren Bedingungen zu tun hat, mag bereits deshalb inadäquat und stossend erscheinen, weil Vorstellungen hierüber immer auch mit Wertauffassungen verbunden sind. Mit der Vernachlässigung dieses Umstandes scheint mir die Problematik solcher Begrifflichkeiten treffender benannt als mit dem Vorwurf, dass die diesen implizit oder explizit zugrunde liegenden Theorien den Ansprüchen wissenschaftlicher Rationalität nicht Genüge leisten. Ich kann mich deshalb auch nicht der Folgerung Herzogs anschliessen, dass es eine erneute Vergewisserung über die «Struktur der (pädagogischen) Wirklichkeit» (S. 4) oder eine neue Bildungstheorie, die in fragwürdiger Weise Empirie und Theorie trennt, ist, die uns in der aktuellen Debatte weiterhilft. «Education», so könnte man vor dem Hintergrund der Standards-Debatte auf der Basis eines breiten Konsenses argumentieren, «has been well defined as initiation into worthwhile activities, and it is clear that assessments of what knowledge (and of what standards) are of most worth, have been, and will continue to be, matters of debate» (Aldrich, 2000, S. 55; Hervorhebungen E.B.). 
Um zu den «Argumenten» zurückzukehren: Diese haben bekanntlich mit Rationalität oder Logik - nicht im Sinn der formalen Logik, sondern im Sinn von richtigen Schlussfolgerungen - zu tun, ein erstes Kriterium, an dem sich auch Herzogs Äusserungen messen lassen müssen ${ }^{1}$. Ebenfalls im Gegensatz zur formalen Logik besteht das Bezugssystem von Argumenten im Feld komplexer sozialer Phänomene selten aus "wahr» und "falsch»; weder ist die «Wahrheit» immer eindeutig, noch ist Erkenntnis abschliessbar. Argumente können unter Umständen endlos gegeneinander ins Feld geführt werden. Eine gewisse Selektivität nach Massgabe der «Triftigkeit» - über die man sich natürlich im Einzelfall streiten kann - bietet sich an, wie überhaupt wissenschaftliche Standards deshalb nicht inexistent sind. Ich will damit sagen: Man kann es bekennenden Gegnern von Bildungsstandards selbstverständlich nicht verwehren, sich in ihren Beweisführungen immer wieder auf kritische Effekte, die sich im Zuge der StandardsBewegung in den USA aufzeigen lassen, zu berufen. Verschiedenes sollte man aber berücksichtigen, wenn man seine Argumente auf entsprechende Literatur abstützt. Erstens existieren inzwischen Recherchen über das dortige Verständnis von Bildungsstandards und Accountability-Systemen, die uns eigentlich eine differenziertere Darstellung und dabei vor allem auch eine Kontextualisierung der Kritiken ermöglichen sollten (Berner \& Stolz, 2006). Zweitens liegen Studien auch zu anderen, europäischen Ländern vor (Döbert, Klieme \& Sroka, 2004; Kotthoff, 2003; van Ackeren, 2003), die es in der Diskussion von Bildungsstandards und Systemen der Qualitätssicherung unbedingt zu berücksichtigen gilt, denn sie liefern bezogen auf den Schweizer Kontext oftmals wertvollere Informationen. Weitet man den Blick auf diese Weise aus, bemerkt man sehr schnell, dass die angewendeten Instrumente (Standards, Tests, Assessments, Accountability) zwar in der Bezeichnung übereinstimmen, dass es aber die zugrunde liegenden (nationalen) Politiken sind, die zu sehr unterschiedlichen Verfahren und Zielsetzungen führen. Insofern ist die Diskussion um Bildungsstandards sicher noch lange nicht zu Ende; es ist jedoch an der Zeit, den Schritt in eine neue Diskussionsphase zu machen, der dem aktuellen Forschungsstand angemessener ist. Insofern sollte man auch die Aussage im von Herzog herangezogenen Motto ernst nehmen: «No reasonable person is against accountability that enhances the quality of education» (Linn, 2003, S. 3). Und weiter heisst es, um den Begriff der geteilten Verantwortung zum Schluss nochmals stark zu machen: «But reasonable people can und do differ regarding what an accountability system needs to entail if it is to help us achieve the shared goal of improved education. It is my contention that, among other things, accountability must entail broadly shared responsibility if it is going to have the positive effects that it is expected to have without having unintended negative effects» (ebd.). 


\section{Anmerkung}

1 So blieb der Autorin der Sinn folgender Schlussfolgerung bis zum Schluss verborgen: «Wenn es wissenschaftliche Erkenntnis ohne theoretische Annahmen nicht gibt, dann kann der Versuch der Indienstnahme der Forschung durch die Politik nur bedeuten, dass von der Theorielosigkeit der Reform des schweizerischen Bildungssystems abgelenkt werden soll» (S. 4; Hervorhebungen E.B.).

\section{Literatur}

Ackeren, I. van (2003). Evaluation, Rückmeldung und Schulentwicklung. Erfahrungen mit zentralen Test, Prüfungen und Inspektionen in England, Frankreich und den Niederlanden. Münster: Waxmann.

Aldrich, R. (2000). Educational Standards in Historical Perspective. In H. Goldstein \& A. Heath (Eds.), Educational Standards (pp. 39-56). Oxford: Oxford University Press.

Berner, E. \& Stolz, St. (2006). Literaturanalyse zu Entwicklung, Anwendung und insbesondere Implementation von Standards in Schulsystemen: Nordamerika. Pädagogisches Institut der Universität Zürich, Fachbereich Allgemeine Pädagogik. Im Auftrag der Schweizerischen Konferenz der kantonalen Erziehungsdirektoren EDK. Zugriff 24.01.08 http://www.edk.ch/d/EDK/Ge-schaefte/framesets/mainHarmoS_d.html

Bloch Pfister, A. (2007). Priester der Volksbildung. Der Professionalisierungsprozess der Zürcher Volksschullehrkräfte zwischen 1770 und 1914. Zürich: Chronos.

Döbert, H., Klieme, E. \& Sroka, W. (Hrsg.). (2004). Conditions of School Performance in Seven Countries. A Quest for Understanding the International Variation of PISA Results. Münster: Waxmann.

Kotthoff, H.-G. (2003). Bessere Schulen durch Evaluation? Internationale Erfahrungen. Münster: Waxmann.

Herzog, W. (2002). Zeitgemässe Erziehung. Die Konstruktion pädagogischer Wirklichkeit. Weilerswist: Velbrück.

Klieme, E., Avenarius, H., Blum, W., Döbrich, P., Gruber, H., Prenzel, M., Reiss, K., Riquarts, K., Rost, J., Ternorth, H.-E. \& Vollmer, H. J. (2003). In Bundesministerium für Bildung und Forschung (Hrsg.), Zur Entwicklung nationaler Bildungsstandards. Eine Expertise. Bonn: BMBF.

Linn, R. L. (2003). Accountability: Responsability and Reasonable Expectations. Educational Researcher, 32 (7), 3-13.

Maradan, O. \& Mangold, M. (2005). Bildungsstandards in der Schweiz. Das Projekt HarmoS. ph akzente, (2) , 3-7.

Oelkers, J. \& Reusser, K. (in Druck, 2008). Qualität entwickeln - Standards sichern - mit Differenz umgehen (S. 211). Expertise im Auftrag des BMBF (Deutschland), BMBWK (Österreich), der EDK (Schweiz) und des MENFP (Luxemburg). Bonn: BMBF.

Seliner-Müller, G., Fries, A.-V. \& Künzli, R. (1999). Wie Lehrpläne entwickelt werden. In R. Künzli, K. Bähr, A.-V.Fries, G. Ghisla, M. Rosenmund \& G. Seliner-Müller (Hrsg.), Lehrplanarbeit. Über den Nutzen von Lehrplänen für die Schule und ihre Entwicklung (S. 31-49). Chur \& Zürich: Rüegger.

Wolf, A. (2000). A Comparative Perspective on Educational Standards. In H. Goldstein \& A. Heath (Eds.), Educational Standards (pp. 9-30). Oxford: Oxford University Press.

Schlagworte: Bildungsreform, Bildungsstandards, Bildungssteuerung, Lehrerprofession, Deprofessionalisierung, Autonomie, Gerechtigkeit 